\title{
Giant Schnauzer
}

National Cancer Institute

\section{Source}

National Cancer Institute. Giant Schnauzer. NCI Thesaurus. Code C53770.

The Giant Schnauzer is a large, compact dog with bushy eyebrows, whiskers and a beard. It has a harsh, wiry outer coat and dense, soft undercoat that comes in solid black or salt and pepper. Height: 23-28 inches (58-71 cm.) Weight: 55-80 pounds (25-36 kg.) 